\title{
ON MEASURES OF COLUMN INDECOMPOSABILITY
}

\author{
D. J. HARTFIEL
}

\begin{abstract}
For any given nonnegative matrix $A$, this paper develops a notion of the $k$ th measure of column indecomposability of $A$. The behavior of this measure on products of matrices is investigated. These results are then applied in developing several results on nonhomogeneous Markov chains.
\end{abstract}

Let $n>1$ be an integer and $N=\{1,2, \ldots, n\}$. Let $A$ be an $n \times n$ nonnegative matrix. If $A$ contains no $r \times s 0$-submatrix with $r+s=n$, then $A$ is said to be fully indecomposable. This notion of full indecomposability arises of ten in the study of nonnegative matrices and has attracted the attention of much research. In [2], this combinatorial notion was quantified by developing a notion of the measure of full indecomposability. This measure is defined as

$$
U_{k}(A)=\min _{|R|+|C|=n-k}\left(\max _{i \in R, j \in C} a_{i j}\right)
$$

for $k=0,1, \ldots, n-2$ with $R, C \subseteq N$ where $S$ denotes the cardinality of the set $S$. Further, let $U_{k}(A)=U_{n-2}(A)$ for $k \geqslant n-2$.

Some use of this quantitative measure was demonstrated in [1], [2], and [3]. This use included the developing of bounds on Perron eigenvalues and eigenvectors of a nonnegative matrix and the development of several results on nonhomogeneous Markov chains.

Let $m \geqslant 1$ be an integer and $M=\{1,2, \ldots, m\}$. The purpose of this paper is to extend the notion of full indecomposability to arbitrary nonnegative matrices and to give a quantitative measure for this extension. For this, let $A$ be an $m \times n$ nonnegative matrix. Set

$$
\mu_{k}=\min _{|R|+|C|=n-k}\left(\max _{i \in R, j \in C} a_{i j}\right)
$$

for $k=0,1, \ldots, n-2$ where $R \subseteq M$ and $C \subseteq N$. Further set $\mu_{k}=\mu_{n-2}$ for

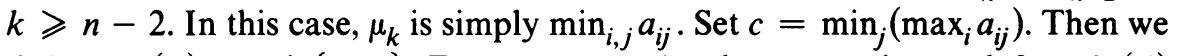
define $C_{k}(A)=\min \left\{c, \mu_{k}\right\}$. For $A$ an $m \times 1$ column matrix we define $C_{k}(A)$

Received by the editors July 7, 1976.

AMS (MOS) subject classifications (1970). Primary 15A48; Secondary 15A51.

Key words and phrases. Indecomposable, measures, nonhomogeneous Markov chains. 
$=\min _{i} a_{i 1}$. If $C_{k}(A)>0$, we say that $A$ is $k$-column indecomposable with $k$ th measure of column indecomposability being $C_{k}(A)$. As examples we have the following.

(a) Let

$$
A=\left(\begin{array}{ll}
1 & 0 \\
1 & 1 \\
0 & 1
\end{array}\right)
$$

Then $C_{0}(A)=0$ and hence $A$ is not 0 -column indecomposable.

(b) Let

$$
A=\left(\begin{array}{lll}
\frac{1}{2} & \frac{1}{3} & 1
\end{array}\right)
$$

Then $C_{0}(A)=\frac{1}{3}$.

(c) Let

$$
A=\left(\begin{array}{cccc}
\frac{1}{3} & \frac{1}{2} & 1 & 0 \\
\frac{1}{2} & 0 & \frac{1}{2} & \frac{1}{4} \\
1 & \frac{1}{4} & 0 & \frac{1}{5}
\end{array}\right)
$$

Then $C_{1}(A)=\frac{1}{5}$.

(d) Let

$$
A=\left(\begin{array}{cccc}
0 & \frac{1}{5} & 0 & 1 \\
\frac{1}{4} & 0 & \frac{1}{2} & 0 \\
\frac{1}{3} & \frac{1}{2} & 0 & \frac{1}{4}
\end{array}\right)
$$

Then $C_{0}(A)=\frac{1}{5}$.

Several properties of $C_{k}$ are immediate.

(1) If $C_{k}(A)>0$, then $A$ has no rows or columns composed of zeros.

(2) $C_{k}(A) \geqslant C_{k+1}(A)$ for $k=0,1, \ldots$

(3) If $A \geqslant B$ then $C_{k}(A) \geqslant C_{k}(B)$ for $k=0,1, \ldots$

(4) $C_{n-2}(A)>0$ if and only if $a_{i j}>0$ for all $i, j$.

(5) If $m=n$ then $C_{k}(A)=U_{k}(A)$.

Thus, from (5) it is seen that $C_{k}$ is a generalization of $U_{k}$ and hence column indecomposable generalizes the notion of full indecomposability. Hence this paper will develop a study of measures of column indecomposability. This study will necessitate some of the technical language in [4]. 
Results. In this section we describe the behavior of $C_{k}$ on products of matrices. The first result in this regard follows.

Lemma. Suppose $A \geqslant 0$ is an $n_{1} \times n_{2}$ matrix which is $k_{1}$-column indecomposable. Suppose $B \geqslant 0$ is an $n_{2} \times n_{3}$ matrix which is $k_{2}$-column indecomposable. Then $A B$ is $k_{1}+k_{2}+1$-column indecomposable.

Proof. The result is obvious for $n_{2}=1$ or $n_{3}=1$, hence we assume that $n_{2} \neq 1$ and $n_{3} \neq 1$. For this, set $A B=C$. Suppose $C$ contains an $r \times s 0$ submatrix, say $C_{2}$. By applying row and column permutations, we may assume, without loss of generality, that

$$
C=\left(\begin{array}{ll}
C_{1} & C_{2} \\
C_{3} & C_{4}
\end{array}\right)
$$

Now, by partitioning $A$ and $B$ we have

$$
\left(\begin{array}{ll}
A_{1} & A_{2} \\
A_{3} & A_{4}
\end{array}\right)\left(\begin{array}{ll}
B_{1} & B_{2} \\
B_{3} & B_{4}
\end{array}\right)=\left(\begin{array}{ll}
C_{1} & C_{2} \\
C_{3} & C_{4}
\end{array}\right)
$$

where $A_{1}$ is $r \times t_{1}, B_{4}$ is $t_{2} \times s$ and $t_{1}+t_{2}=n_{2}$. Again by simultaneous column and row permutations on $A$ and $B$ respectively, we may assume that $t_{1}$ was chosen so that $A_{1}$ has no column of zeros and $A_{2}=0$. Now $A_{1} B_{2}+A_{2} B_{4}=0$ implies $B_{2}=0$. As $B$ has no column of zeros it follows that $B_{4} \neq 0$.

Now, $r+t_{2}<n_{2}-k_{1}$ and $t_{1}+s<n_{3}-k_{2}$. Thus $r<t_{1}-k_{1}$ and hence $r+k_{1}+s+1<n_{3}-k_{2}$ or $r+s<n_{3}-\left(k_{1}+k_{2}+1\right)$. Hence, as $C_{2}$ was arbitrary, $C$ is $k_{1}+k_{2}+1$-column indecomposable.

Corollary 1. Suppose $A \geqslant 0$ is an $n_{1} \times n_{2}$ matrix and $B \geqslant 0$ is an $n_{2} \times n_{3}$ matrix with $C_{k_{1}}(A)>0$ and $C_{k_{2}}(B)>0$. Then

$$
C_{k_{1}+k_{2}+1}(A B) \geqslant C_{k_{1}}(A) C_{k_{2}}(B) \text {. }
$$

Proof. Let

$$
\bar{A}=\left(\bar{a}_{i j}\right) \quad \text { where } \bar{a}_{i j}= \begin{cases}a_{i j} & \text { if } a_{i j} \geqslant C_{k_{1}}(A), \\ 0 & \text { otherwise }\end{cases}
$$

and

$$
\bar{B}=\left(\bar{b}_{i j}\right) \quad \text { where } \bar{b}_{i j}= \begin{cases}b_{i j} & \text { if } b_{i j} \geqslant C_{k_{2}}(B), \\ 0 & \text { otherwise }\end{cases}
$$

Thus, $C_{k_{1}}(A)=C_{k_{1}}(\bar{A})$ and $C_{k_{2}}(B)=C_{k_{2}}(\bar{B})$.

Now, as $A B \geqslant \bar{A} \bar{B}$ it follows that $C_{k_{1}+k_{2}+1}(A B) \geqslant C_{k_{1}+k_{2}+1}(\bar{A} \bar{B})$. As each 
nonzero entry of $\bar{A} \bar{B}$ is greater than or equal to $C_{k_{1}}(\bar{A}) C_{k_{2}}(\bar{B})$ it follows from the lemma that $C_{k_{1}+k_{2}+1}(\bar{A} \bar{B}) \geqslant C_{k_{1}}(\bar{A}) C_{k_{2}}(\bar{B})$. Thus,

$$
C_{k_{1}+k_{2}+1}(A B) \geqslant C_{k_{1}}(A) C_{k_{2}}(B) \text {. }
$$

By induction, this corollary can be extended as follows.

Corollary 2. Suppose $A_{i} \geqslant 0$ is an $n_{i} \times n_{i+1}$ matrix and $C_{k_{i}}\left(A_{i}\right)>0$ for $i=1,2, \ldots, r$. Then

$$
C_{k_{1}+k_{2}+\cdots+k_{r}+r-1}\left(A_{1} A_{2} \cdots A_{r}\right) \geqslant C_{k_{1}}\left(A_{1}\right) C_{k_{2}}\left(A_{2}\right) \cdots C_{k_{r}}\left(A_{r}\right) .
$$

On positive products of matrices we add the following.

COROllary 3. Suppose $A_{i} \geqslant 0$ is an $n_{i} \times n_{i+1}$ matrix and $C_{k_{i}}\left(A_{i}\right)>0$ for $i=1,2, \ldots, r$ with $k_{1}+k_{2}+\cdots+k_{r}+r+1 \geqslant n_{r}$. Then $B=A_{1} A_{2} \cdots A_{r}$ $>0$ and $b_{i j} \geqslant C_{k_{1}}\left(A_{1}\right) C_{k_{2}}\left(A_{2}\right) \cdots C_{k_{r}}\left(A_{r}\right)$.

Proof. The proof follows by noting that $k_{1}+k_{2}+\cdots+k_{r}+r+1 \geqslant n_{r}$ implies that $k_{1}+k_{2}+\cdots+k_{r}+r-1 \geqslant n_{r}-2$. Thus by the definition of $C_{n_{r}-2}$ we have for every element $b_{i j}$ of $B$ that

$$
\begin{aligned}
b_{i j} & \geqslant C_{n_{r}-2}(B) \geqslant C_{k_{1}+k_{2}+\cdots+k_{r}+r+1}(B) \\
& \geqslant C_{k_{1}}\left(A_{1}\right) C_{k_{2}}\left(A_{2}\right) \cdots C_{k_{r}}\left(A_{r}\right)
\end{aligned}
$$

which proves the corollary.

As a direct consequence of this corollary we have the following.

COROllary 4. Suppose $A_{i} \geqslant 0$ is an $n_{i} \times n_{i+1}$ matrix and $C_{k_{i}}\left(A_{i}\right)>0$ for $i=1,2, \ldots$ with $\max _{i} n_{i} \leqslant n$. Let $r$ be any positive integer so that $r \geqslant n-1$. Then $B=A_{s+1} A_{s+2} \cdots A_{s+r}>0$ and

$$
b_{i j} \geqslant C_{k_{s+1}}\left(A_{s+1}\right) C_{k_{s+2}}\left(A_{s+2}\right) \cdots C_{k_{s+r}}\left(A_{s+r}\right)
$$

for any $s \geqslant 0$.

This then concludes the initial results of the paper. The significance of these results is the topic for the next section.

Applications. The basic purpose of this section is to show the use of $C_{k}$ in the study of nonhomogeneous Markov chains. A first result in this regard follows.

THEOREM 1. Suppose $A_{i}$ is an $n_{i} \times n_{i+1}$ stochastic matrix with $C_{0}\left(A_{i}\right) \geqslant \mu$ $>0$ and $n_{i} \leqslant n$ for $i=1,2, \ldots$ Then $A_{1}, A_{2}, \ldots$ is weakly ergodic.

Proof. By Corollary 4 , there is a positive integer $r$ so that $A_{s+1} A_{s+2} \cdots A_{s+r}$ 
$=P_{s, r}>0$ with $p_{i j}^{(s, r)} \geqslant \mu^{r}$ for all $i$ and $j$. Let $x \geqslant 0$ be an $n_{s+r}$-dimensional vector with $\min _{i} x_{i}=\underline{x}$ and $\max _{i} x_{i}=\bar{x}$. Then, by considering the components of $P_{s, r} x=y$ it follows that

$$
\left(1-\mu^{r}\right) \underline{x}+\mu^{r} \bar{x} \leqslant \sum_{k=1}^{n_{s+r}} p_{i k}^{(s, r)} x_{k} \leqslant \mu^{r} \underline{x}+\left(1-\mu^{r}\right) \bar{x}
$$

for all $i$, so

$$
\max _{i, j}\left|y_{i}-y_{j}\right| \leqslant\left(1-2 \mu^{r}\right)(\bar{x}-\underline{x}) .
$$

Applying this result to $P_{s, r} P_{s+r, r}=B$ it follows that

$$
\max _{i, j, k}\left|b_{i k}-b_{j k}\right| \leqslant\left(1-2 \mu^{r}\right) \max _{i, j, k}\left|p_{i k}^{(s+r, r)}-p_{j k}^{(s+r, r)}\right| .
$$

Thus, by induction, if $P_{s, r} P_{s+r, r} \cdots P_{s+k r, r}=B$ then

$$
\max _{i, j, k}\left|b_{i k}-b_{j k}\right| \leqslant\left(1-2 \mu^{r}\right)^{k} \max _{i, j, k}\left|p_{i k}^{(s+k r, r)}-p_{j k}^{(s+k r, r)}\right| \leqslant\left(1-2 \mu^{r}\right)^{k} .
$$

Hence, $P_{s, r}, P_{s+r, r}, \ldots$ is a weakly ergodic Markov chain for any $s \geqslant 0$ and thus $A_{1}, A_{2}, \ldots$ is a weakly ergodic Markov chain.

Strongly ergodic nonhomogeneous Markov chain results are not possible for the general problem, although results of this type can be obtained for special cases. A special case of interest requires the following definition.

Let $A_{i}$ be an $n_{i} \times n_{i+1}$ stochastic matrix for $i=1,2, \ldots$ The sequence $A_{1}, A_{2}, \ldots$ will be called a state cyclic chain if and only if there is a positive integer $d$ so that $n_{k}=n_{k+d}$ for all $k$. The integer $d$ is called the length of the state cyclic chain.

It is the intent now to show that a strongly ergodic type result can be obtained for state cyclic chains. For this we have the following.

TheOREM 2. Suppose $A_{1}, A_{2}, \ldots$ is a state cyclic chain having length $d$. If $\operatorname{limit}_{k \rightarrow \infty} A_{m+d k}=B_{m}$ and $C_{0}\left(B_{m}\right)>0(m \bmod d)$ then

$$
\operatorname{limit}_{k \rightarrow \infty} A_{1} A_{2} \cdots A_{m+d k}=A_{1} \cdots A_{m}\left(B_{m+1} B_{m+2} \cdots B_{m-1} B_{m}\right)^{\infty} \text {. }
$$

Proof. Consider the Markov chain $A_{m+1}, A_{m+2}, \ldots$ Let

$$
P_{1}=A_{m+1} \cdots A_{m+d}, \quad P_{2}=A_{m+d+1} \cdots A_{m+2 d}, \cdots
$$

Then $P$ is $n_{m} \times n_{m}$ for all $i$. Further, by property (3) and Corollary 2,

$$
\begin{aligned}
U_{0}\left(B_{m+1} B_{m+2} \cdots B_{m-1} B_{m}\right) & =C_{0}\left(B_{m+1} B_{m+2} \cdots B_{m-1} B_{m}\right) \\
& \geqslant C_{0}\left(B_{m+1}\right) C_{0}\left(B_{m+2}\right) \cdots C_{0}\left(B_{m-1}\right) C_{0}\left(B_{m}\right) .
\end{aligned}
$$


Now, by Lemma 3 in [3], limit ${ }_{k \rightarrow \infty} P_{1} P_{2} \cdots P_{k}=\left(B_{m+1} B_{m+2} \cdots B_{m-1} B_{m}\right)^{\infty}$. Finally, then,

$$
\begin{aligned}
\operatorname{limit}_{k \rightarrow \infty} A_{1} A_{2} \cdots A_{d k+m} & =\operatorname{limit}_{k \rightarrow \infty}\left(A_{1} \cdots A_{m}\right) P_{1} P_{2} \cdots P_{k} \\
& =A_{1} \cdots A_{m}\left(B_{m+1} B_{m+2} \cdots B_{m-1} B_{m}\right)^{\infty}
\end{aligned}
$$

which establishes the theorem.

To help in the understanding of the nature of this theorem, an example is included.

EXAmple. Consider the following state cyclic chains.

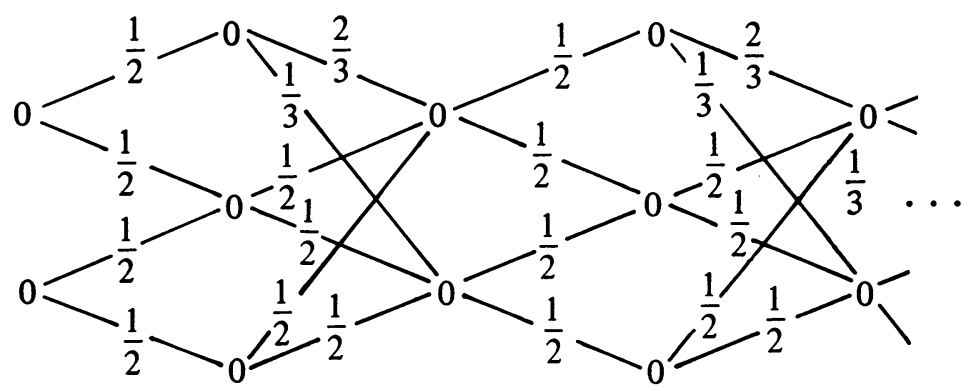

Let

$$
A=\left(\begin{array}{lll}
\frac{1}{2} & \frac{1}{2} & 0 \\
0 & \frac{1}{2} & \frac{1}{2}
\end{array}\right)
$$

and

$$
B=\left(\begin{array}{ll}
\frac{2}{3} & \frac{1}{3} \\
\frac{1}{2} & \frac{1}{2} \\
\frac{1}{2} & \frac{1}{2}
\end{array}\right)
$$

Then $A_{2 k-1}=A$ and $A_{2 k}=B$ for $k=1,2, \ldots$ By direct calculation, $C_{0}(A)>0$ and $C_{0}(B)>0$. Further, $d=2$ with

$$
A B=\left(\begin{array}{cc}
\frac{7}{12} & \frac{5}{12} \\
\frac{1}{2} & \frac{1}{2}
\end{array}\right)
$$

and 


$$
B A=\left(\begin{array}{ccc}
\frac{2}{6} & \frac{1}{2} & \frac{1}{6} \\
\frac{1}{4} & \frac{1}{2} & \frac{1}{4} \\
\frac{1}{4} & \frac{1}{2} & \frac{1}{4}
\end{array}\right)
$$

Thus,

$$
(A B)^{\infty}=\left(\begin{array}{cc}
\frac{6}{11} & \frac{5}{11} \\
\frac{6}{11} & \frac{5}{11}
\end{array}\right), \quad(B A)^{\infty}=\left(\begin{array}{ccc}
\frac{3}{11} & \frac{1}{2} & \frac{5}{22} \\
\frac{3}{11} & \frac{1}{2} & \frac{5}{22} \\
\frac{3}{11} & \frac{1}{2} & \frac{5}{22}
\end{array}\right)
$$

and

$$
A_{1}(B A)=\left(\begin{array}{ccc}
\frac{3}{11} & \frac{1}{2} & \frac{5}{22} \\
\frac{3}{11} & \frac{1}{2} & \frac{5}{22}
\end{array}\right)
$$

Hence, if we let $y_{1}^{k}, y_{2}^{k}, y_{3}^{k}$ denote the probabilities of being in state 1 , state 2 , and state 3 respectively at time $k$, then, in the long run, $y_{1}^{k}$ approaches $\frac{6}{11}, y_{2}^{k}$ approaches $\frac{5}{11}$ for $k$ even and $y_{1}^{k}$ approaches $\frac{3}{11}, y_{2}^{k}$ approaches $\frac{1}{2}, y_{3}^{k}$ approaches $\frac{5}{22}$ for $k$ odd.

Using the notion of state cyclic chains, it is also possible to develop a type of mean transition probability result. For this we have the following.

TheOREM 3. Suppose $A_{1}, A_{2}, \ldots$ is a state cyclic chain having length $d$. Suppose limit ${ }_{k \rightarrow \infty} A_{m+d k}=B_{m}$ and $C_{0}\left(B_{m}\right)>0(m \bmod d)$. Then

$$
\begin{gathered}
\operatorname{limit}_{k \rightarrow \infty} \frac{A_{1} A_{2} \cdots A_{m+d}+A_{1} A_{2} \cdots A_{m+2 d}+\cdots+A_{1} A_{2} \cdots A_{m+k d}}{k} \\
=A_{1} A_{2} \cdots A_{m}\left(B_{m+1} B_{m+2} \cdots B_{m}\right)^{\infty}
\end{gathered}
$$

for all $m, 0 \leqslant m<d$.

Proof. As $C_{0}\left(B_{m}\right)>0(m \bmod d)$, it follows from property (3) and Corollary 2 that

$$
\begin{aligned}
U_{0}\left(B_{s+1} B_{s+2} \cdots B_{s+d}\right) & =C_{0}\left(B_{s+1} B_{s+2} \cdots B_{s+d}\right) \\
& \geqslant C_{0}\left(B_{s+2}\right) \cdots C_{0}\left(B_{s+d}\right)>0,
\end{aligned}
$$

for any positive integer $s,(s+k \bmod d$ for all $s$ and $k)$. Thus, as 


$$
\operatorname{limit}_{k \rightarrow \infty} A_{m+1+k d} \cdots A_{m+(k+1) d}=B_{m+1} B_{m+2} \cdots B_{m}
$$

it follows from Theorem 1 of [3] that

$$
\begin{gathered}
\operatorname{limit}_{k \rightarrow \infty} \frac{A_{m+1} A_{m+d}}{+A_{m+1} \cdots A_{m+2 d}+\cdots+A_{m+1} \cdots A_{m+k d}} \\
k \\
=\left(B_{m+1} B_{m+2} \cdots B_{m-1}\right)^{\infty} .
\end{gathered}
$$

Thus

$$
\begin{gathered}
\operatorname{limit}_{k \rightarrow \infty} \frac{A_{1} A_{2} \cdots A_{m+d}+A_{1} A_{2} \cdots A_{m+2 d}+\cdots+A_{1} A_{2} \cdots A_{m+k d}}{k} \\
=A_{1} A_{2} \cdots A_{m}\left(B_{m+1} B_{m+2} \cdots B_{m-1}\right)^{\infty}
\end{gathered}
$$

As a consequence of this theorem we have the following.

Corollary 5. Suppose $A_{1}, A_{2}, \ldots$ is a state cyclic chain of length $d>1$, and of length $d^{\prime}$ where $d^{\prime} r=d$ for some integer $r$. Suppose limit ${ }_{k \rightarrow \infty} A_{m+d k}=B_{m}$, and $C_{0}\left(B_{m}\right)>0(m \bmod d)$. Then

$$
\begin{aligned}
& \operatorname{limit}_{k \rightarrow \infty} \frac{A_{1} A_{2} \cdots A_{m+d^{\prime}}+A_{1} A_{2} \cdots A_{m+2 d^{\prime}}+\cdots+A_{1} A_{2} \cdots A_{m+k d^{\prime}}}{k} \\
&=\frac{1}{r}\left[A_{1} A_{2} \cdots A_{m}\left(B_{m+1} \cdots B_{m+d}\right)^{\infty}+A_{1} A_{2} \cdots A_{m+d^{\prime}}\left(B_{m+d^{\prime}+1} B_{m+d^{\prime}+d}\right)^{\infty}\right. \\
&\left.\quad+\cdots+A_{1} A_{2} \cdots A_{m+(r-1) d^{\prime}}\left(B_{m+(r-1) d^{\prime}+1} \cdots B_{m+(r-1) d^{\prime}+d}\right)^{\infty}\right]
\end{aligned}
$$

for all $m, 0 \leqslant m<d^{\prime}$

Proof. Pick $m, 0 \leqslant m<d^{\prime}$ and $t, 0 \leqslant t<r$. Then by Theorem 3,

$$
\begin{gathered}
\operatorname{limit}_{k \rightarrow \infty} \frac{A_{1} A_{2} \cdots A_{m+t d^{\prime}+d}+A_{1} A_{2} \cdots A_{m+t d^{\prime}+2 d}+\cdots+A_{1} A_{2} \cdots A_{m+t d^{\prime}+k d}}{k} \\
=A_{1} A_{2} \cdots A_{m+t d^{\prime}}\left(B_{m+t d^{\prime}+1} B_{m+t d^{\prime}+2} \cdots B_{m+t d^{\prime}+d}\right)^{\infty} .
\end{gathered}
$$

Summing these equations for all $t, 0 \leqslant t<r$, and dividing by $r$ yields the result.

As an aid to the understanding of this corollary we provide the following elementary example.

Example. Let $A=\left(\frac{1}{2} \frac{1}{2}\right), B=\left(\begin{array}{l}1 \\ 1\end{array}\right), C=\left(\begin{array}{ll}\frac{1}{3} & \frac{2}{3}\end{array}\right)$ and $D=\left(\begin{array}{l}1 \\ 1\end{array}\right)$. Let $A_{1+4 k}=$ $A, A_{2+4 k}=B, A_{3+4 k}=C$ and $A_{4+4 k}=D$ for $k=0,1,2, \ldots$ Then $A_{1}, A_{2}, \ldots$ is a state cyclic chain with length $d=4$ and length $d^{\prime}=2$. Thus from the corollary, 


$$
\begin{aligned}
\operatorname{limit}_{k \rightarrow \infty} \frac{A_{1}+A_{1} A_{2} A_{3}+\cdots+A_{1} A_{2} \cdots A_{2 k-1}}{k} \\
=\frac{1}{2}\left[\left(\frac{1}{2} \frac{1}{2}\right)+\left(\frac{1}{3} \frac{2}{3}\right)\right]=\left(\frac{5}{12} \frac{7}{12}\right),
\end{aligned}
$$

and

$$
\begin{gathered}
\operatorname{limit}_{k \rightarrow \infty} \frac{A_{1} A_{2}+A_{1} A_{2} A_{3} A_{4}+\cdots+A_{1} A_{2} \cdots A_{2 k}}{k} \\
=\frac{1}{2}[(1)+(1)]=(1) .
\end{gathered}
$$

Hence, if we consider mean probabilities of the odd states and the mean probability of the even states, then, in the long run, the mean of the odd states approaches $\left(\frac{5}{12} \frac{7}{12}\right)$ while the mean of even states is 1 .

From these results then it is seen that $C_{k}$ is a useful tool in the study of nonhomogeneous Markov chains. Other applications are no doubt also possible.

\section{REFERENCES}

1. D. J. Hartfiel, A result concerning strongly ergodic nonhomogenuous Markov chains, Linear Algebra and Appl. 9 (1974), 169-174. MR 50 \# 11457.

, Results on measures of irreducibility and full indecomposability, Trans. Amer. Math. Soc. 202 (1975), 357-368. MR 51 \#558.

3. _ Two theorems generalizing the mean transition probability results in the theory of Markov chains, Linear Algebra and Appl. 11 (1975), no. 2, 181-187. MR 51 \# 10372.

4. E. Seneta, Non-negative matrices, Wiley, New York, 1973.

Department of Mathematics, Tryas a\&M Univirsity, Collegl Station, Texas 77843 